\title{
Adsorption properties of methylene blue on hectorite: kinetic and Thermodynamics studies
}

\author{
Jun $M A^{1, a}$, Su-yan $\operatorname{TIAN}^{1, b}$, Shan-liang $M U^{1, b}$, Shao-lei XIE ${ }^{2, d}$, Rong-jian \\ $\mathrm{YING}^{1, \mathrm{e}}$ \\ ${ }^{1}$ School of Chemistry and Chemical Engineering, Linyi University, 276000, China \\ ${ }^{2}$ Department of Electrical Engineering and Automation, Luoyang Institute of Science and \\ Technology, Luoyang, 471023, China \\ amajun716@163.com, ${ }^{b}$ tianyan198289@163.com, ${ }^{c}$ chem@lyu.edu.cn, ${ }^{d} x$ sl2013@isl.ac.cn, \\ eyingrongjian@lyu.edu.cn \\ * Su-yan TIAN
}

Keywords: Hectorite, methylene blue, kinetic, thermodynamics

\begin{abstract}
Hectorite has been designed and synthesized by hydrothermal way, which is used for removal of methylene blue (MB) from aqueous solution. The experiment data was analyzed using pseudo-second-order kinetics, intra-particle diffusion and film diffusion. The characteristic parameters for each model have been determined. Thermodynamic parameters such as activation $\left(E_{a}\right)$, standard free energy $\left(\Delta G^{0}\right)$, the enthalpy $\left(\Delta H^{0}\right)$ and the entropy $\left(\Delta S^{0}\right)$ were also evaluated. The negative value of $\Delta G^{0}(\mathrm{~kJ} / \mathrm{mol})$ and $\Delta H^{0}(\mathrm{~kJ} / \mathrm{mol})$ indicates the spontaneous and exothermic nature of the reactions. The data of $E_{a}$ shows the physisorption process of $\mathrm{MB}$ on hectorite.
\end{abstract}

\section{Introduction}

The main pollution source of textile effluent emerged from the dyeing process. The effluent discharged was highly colored. The disposal of this colored water was not only caused damage to aquatic life, but also to human beings by causing mutagenic or carcinogenic effect[1]. Accordingly, to protect humans and recover ecosystem from contamination, the dyes must be eliminated from the dye-contained wastewater before being released into the environment. Therefore, the removal of dyes from waste effluents was important. During the past three decades, different methods such as coagulation, electro coagulation, flotation, chemical oxidation, filtration, membrane separation and microbial degradation have been developed for the removal of synthetic dyes from waters and wastewaters to decrease its impact in the environment [2-4]. Among the numerous techniques of dye removal, adsorption is the procedure of choice giving the best results as it can be used to remove certain classes of chemical pollutants from water. Conventional industries used activated carbon as adsorbent for wastewater treatment. Because of its high cost, researchers are testing the efficiency of various other low cost adsorbents for the textile effluents. A lot of works have employed natural and synthetic clays for removing the dyes from wastewater [5-7].

Hectorite, which is 2:1 smectite clay, has been used as an adsorbent for the removal of cationic dyes and metal ions [8-10]. Due to unique structure with interior channels which allows penetration of organic and inorganic ions bounding together with solutes into the structure of hectorite, it is one of the best adsorbents for removing dyes from relatively concentrated wastewater. However, the adsorption processes of $\mathrm{MB}$ onto hectorite such as equilibrium model, dynamics, and thermodynamic were few reported .

In this paper, using hydrothermal way, we synthesized hectorite and proposed the use of hectorite for the adsorption of methylene blue(MB). Experiment data was analyzed using kinetic and thermodynamics equations. The characteristic parameters for each model have been determined. 


\section{Experimental procedures}

\section{Adsorption experiments.}

All adsorption experiments were carried out in a thermostatic orbital shaker at different temperatures (293, 303, 313 and 323K). The shaking was continued till the equilibrium time was reached. The adsorption isotherm experiment was carried out by agitating aqueous dispersions of MB (200 $\mathrm{mL}$, various concentrations) and absorbent $(100 \mathrm{mg})$. The dispersion was centrifuged and the concentration was measured by UV-Vis spectroscopy (T-6 UV-VIS spectrophotometer) at 665 $\mathrm{nm}$. The amount of MB adsorbed by hectorites, qe $(\mathrm{mg} / \mathrm{g})$, was calculated by the following mass balance relationship:

$$
q_{e}=\left(C_{0}-C_{e}\right) \frac{v}{w}
$$

Where $C_{0}$ and $C_{e}$ are the initial and equilibrium solution concentrations of $\mathrm{MB}$, respectively $(\mathrm{mg} / \mathrm{L}), \mathrm{v}$ is the volume of the solution $(L)$ and $\mathrm{w}$ is the mass $(\mathrm{g})$ of the adsorbent used.

The kinetic studies were carried out in $200 \mathrm{~mL} \mathrm{MB}$ solutions $(180 \mathrm{mg} / \mathrm{L})$ by adding $100 \mathrm{mg}$ adsorbent at different temperatures (293, 303, 313 and 323K). At given irradiation time intervals, the dispersion was centrifuged and the concentration was measured.

\section{Results and discussion}

\section{The pseudo-second-order kinetic model.}

The study of adsorption dynamics described the solute adsorption rate. This rate controlled the residence time of adsorbate adsorption onto hectorite was analyzed using pseudo-first-order, pseudo-second-order, intra-particle diffusion kinetics models.

The amount of MB adsorbed onto hectorite increased as the time increased. Most of MB molecules adsorption took place at the initial $30 \mathrm{~min}$ of the experiment. As the time progressed, there was a weak increase in adsorption even after long time.

The linear form of the pseudo-second-order kinetic model [11] was given as,

$$
\frac{t}{q_{t}}=\frac{1}{k_{2} q_{e}^{2}}+\frac{t}{q_{e}}
$$

where $k_{2}$ was the equilibrium rate constant of pseudo-second-order adsorption $[\mathrm{g} /(\mathrm{mg} \cdot \mathrm{min})]$. The straight line plots of $t / q t$ against $t$ were tested to obtain rate parameters (Fig. 1 and Table 1).

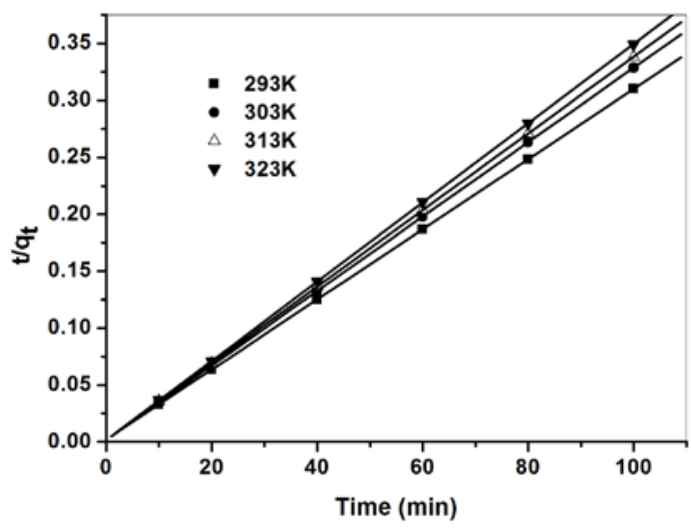

Fig. 1 The pseudo-second-order kinetics for the adsorption of MB onto hectorite

The correlation coefficients $\left(r^{2}\right)$ of the pseudo-second-order model were higher (Table 1$)$. The experimental $q_{e}$ values studied for hectorite were close to $q_{e}$ values calculated from the pseudo-second-order kinetic model. It could be concluded that the mechanism of adsorption was pseudo-second-order reaction. 
Table 1 The kinetic parameter constants for the adsorption MB onto hectorite

\begin{tabular}{ccccc}
\hline Adsorption & \multirow{2}{*}{$\begin{array}{c}\text { Experimental } \\
\text { qe }(\mathrm{mg} / \mathrm{g})\end{array}$} & \multicolumn{3}{c}{ Pseudo-second-order kinetic model } \\
\cline { 3 - 5 } & 338 & $\begin{array}{c}\mathrm{q}_{\mathrm{e}} \\
(\mathrm{mg} / \mathrm{g})\end{array}$ & $k_{2}$ & $\mathrm{r}_{2}{ }^{2}$ \\
\hline $293 \mathrm{~K}$ & 330 & 325 & 0.0048 & 0.9994 \\
$303 \mathrm{~K}$ & 317 & 306 & 0.0063 & 0.9996 \\
$313 \mathrm{~K}$ & 301 & 297 & 0.0067 & 0.9995 \\
$323 \mathrm{~K}$ & 287 & 0.0078 & 0.9998 \\
\hline
\end{tabular}

\section{Intraparticle diffusion and film diffusion kinetics.}

Intraparticle model used here referred to the theory proposed by Weber and Morris. The linear form of intraparticle diffusion model was given as,

$$
q_{t}=K_{P} t^{1 / 2}+C
$$

where $C$ was the intercept and $K_{P}$ was the intra-particle diffusion rate constant $\left[\mathrm{mg} /\left(\mathrm{g} \cdot \mathrm{min}^{1 / 2}\right)\right]$. According to this model, plot of $q_{t}$ versus $t^{1 / 2}$ should be linear if intra-particle diffusion was involved in the adsorption process [11]. If these lines passed through the origin, then intra-particle diffusion was the rate controlling step. When these lines did not pass through the origin, this was indicative of some degree of boundary layer control and this further showed that the intra-particle diffusion was not the only rate limiting step. The values of $K_{P}$ and $C$ were given in Table 2. In this experiment, the plots did not pass through the origin, which indicated the intra-particle diffusion was not only the rate controlling step. The result was consistent with the previous study [12].

If the film diffusion was one of the rate limiting steps, the calculated film diffusion coefficient $\left(D_{F}\right)$ value will be in the rage $10^{-6}$ to $10^{-8} \mathrm{~cm}^{2} / \mathrm{s}$. The film diffusion coefficient $\left(D_{F}\right)$ values were calculated using the following equation

$$
D_{F}=\left(0.23 r_{0} \delta C_{S}\right) /\left(C_{L} t_{0.5}\right)
$$

where $r_{0}$ and $\mathrm{t}^{0.5}$ have the same meaning as above, $\delta$ is the film thickness $\left(10^{-3} \mathrm{~cm}\right)$ [13], $C_{S}$ and $C_{L}$ are the concentrations of adsorbate in solid and liquid phase at $\mathrm{t}=\mathrm{t}$ and $\mathrm{t}=0$, respectively. The calculated $D_{F}$ value are found to be in the order of $10^{-8} \mathrm{~cm}^{2} / \mathrm{s}$ (Table 2), which implied the film diffusion was one of the rate limiting steps.

Based on all the explanation, the pseudo-second-order model was the best fit model for the adsorption of MB onto cobalt doping hectorite. The adsorption process was controlled by both intra-particle diffusion and film diffusion. The initial section indicated the external surface adsorption. The second indicated the final equilibrium stage where the intra-particle diffusion starts to slow down due to extremely low solute concentrations in the solution.

Table 2 Intra-particle diffusion model parameter and film diffusion coefficient for the adsorption of MB onto hectorite at different temperature

\begin{tabular}{ccccc}
\hline \multirow{2}{*}{ Adsorption } & \multicolumn{3}{c}{ Intraparticle diffusion model } & Film diffusion coefficient \\
\cline { 2 - 4 } & $\mathrm{K}_{\mathrm{P}}$ & $C(\mathrm{mg} / \mathrm{g})$ & $\mathrm{r}_{\mathrm{P}}{ }^{2}$ & $\begin{array}{c}\left(\mathrm{D}_{\mathrm{F}}\right) \\
\left(\mathrm{cm}^{2} / \mathrm{s}\right)\end{array}$ \\
\hline 293K & 1.3121 & 311 & 0.8951 & $1.4792 \times 10^{-8}$ \\
$303 \mathrm{~K}$ & 1.0300 & 295 & 0.8946 & $1.7211 \times 10^{-8}$ \\
$313 \mathrm{~K}$ & 0.81735 & 288 & 0.8941 & $1.7243 \times 10^{-8}$ \\
$323 \mathrm{~K}$ & 0.6110 & 280 & 0.9748 & $1.8745 \times 10^{-8}$ \\
\hline
\end{tabular}

Thermodynamics studies.

The pseudo-second-order rate constant of $\mathrm{MB}$ adsorption was expressed as a function of temperature by Arrhenius type relationship

$$
\ln k_{2}=-\frac{E_{a}}{R T}+\ln A
$$

where $E_{a}$ is the Arrhenius activation energy of sorption, representing the minimum energy which reactants must have to proceed, $\mathrm{A}$ is the Arrhenius factor, $\mathrm{R}$ is the gas constant and is equal to 8.314 
$\mathrm{J} / \mathrm{mol} \cdot \mathrm{K}$, and $\mathrm{T}$ is the solution temperature. When Ink is plotted vs $1 / \mathrm{T}$ (Fig.2), a straight line with slope $-E_{a} / R$ is obtained. The magnitude of activation energy gives an idea about the type adsorption, which is mainly physical or chemical. The physisorption processes usually have energies in the range of 5-40 kJ/mol, while higher activation energies (40-800 $\mathrm{kJ} / \mathrm{mol})$ suggest chemisorptions [14]. The result obtained is $12.00 \mathrm{~kJ} / \mathrm{mol}$, which are in the typical activation energy range for physisorption. This conclusion was also inferred from the dispersive interaction between MB and surface of hectorite.

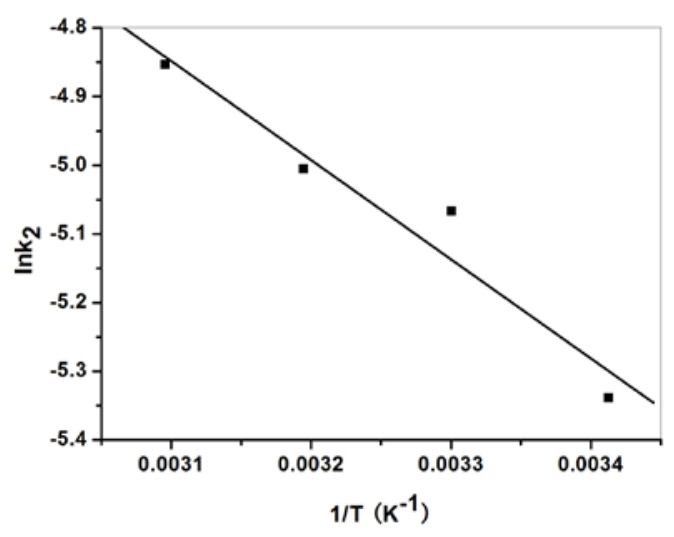

Fig.2 Arrhenius plots for the adsorption of MB onto hectorite

The thermodynamic parameters such as change in standard free energy $\left(\Delta G^{0}\right)$, enthalpy $\left(\Delta H^{0}\right)$ and entropy $\left(\triangle S^{0}\right)$ were determined by using the following equations and were given in Table 3 .

$$
\begin{aligned}
& K=\frac{C_{A}}{C_{S}} \\
& \Delta G^{o}=-R T \ln K \\
& \ln K=-\frac{\Delta H^{o}}{R T}+\frac{\Delta S^{o}}{R}
\end{aligned}
$$

where $K$ was the equilibrium constant, $C_{A}$ was the amount of MB adsorbed on the adsorbent at equilibrium $(\mathrm{mg} / \mathrm{g})$, and $C_{S}$ was the equilibrium concentration $(\mathrm{mg} / \mathrm{L})$ of the MB in the solution. $T$ was the solution temperature $(K)$ and $\mathrm{R}$ was the gas constant.

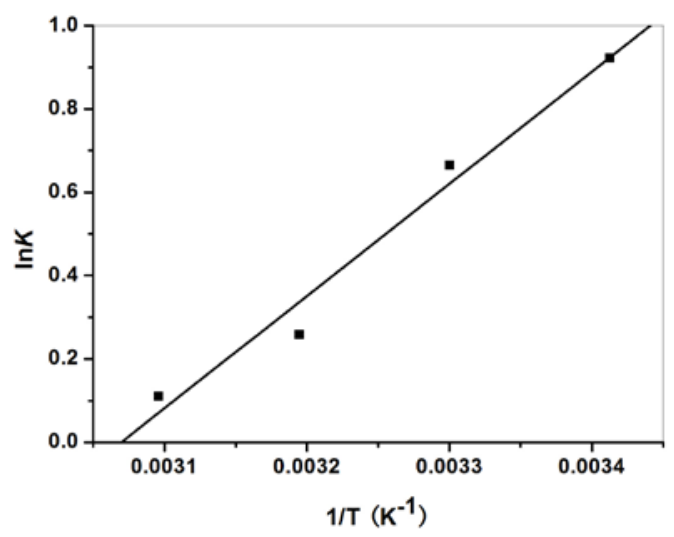

Fig.3 Plot of $\ln K$ vs. $1 / T$ for MB onto hetorite. $K$ is distribution coefficient, $T$ is temperature 293k, 303K, 313K, 323K

Table 3 Thermodynamic parameters for the adsorption of MB onto hectorite

\begin{tabular}{cccccc}
\hline Adsorption & $K$ & $\begin{array}{c}\mathrm{Ea} \\
\left(\mathrm{kJ} \cdot \mathrm{mol}^{-1}\right)\end{array}$ & $\begin{array}{c}\Delta G^{o} \\
\left(\mathrm{~kJ} \cdot \mathrm{mol}^{-1}\right)\end{array}$ & $\begin{array}{c}\Delta H^{o} \\
\left(\mathrm{~kJ} \cdot \mathrm{mol}^{-1}\right)\end{array}$ & $\begin{array}{c}\Delta S^{o} \\
\left(\mathrm{~J} \cdot \mathrm{K}^{-1} \mathrm{~mol}^{-1}\right)\end{array}$ \\
\hline 293K & 2.5128 & & -2.245 & & \\
$303 \mathrm{~K}$ & 1.9429 & 12.00 & -1.673 & -22.39 & -68.74 \\
$313 \mathrm{~K}$ & 1.2931 & & -0.669 & & \\
323K & 1.1165 & & -0.296 & & \\
\hline
\end{tabular}


$\Delta H^{0}$ and $\Delta S^{0}$ were determined from the slope and intercept of van't Hoff plot of $\ln K$ versus $1 / T$, which were shown in Fig. 3 and Table 2.The obtained values of $\Delta H^{0}$ and $\Delta \mathrm{S}^{0}$ are $-22.39 \mathrm{~kJ} / \mathrm{mol}$ and $-68.74 \mathrm{~J} / \mathrm{mol} \cdot \mathrm{K}$, respectively. It means that adsorption of MB on hectorite is an exothermic process, so raising the temperature leads to lower affinity and less MB being adsorbed at equilibrium.

\section{Conclusion}

Hectorite was synthesized successfully. With large pore volumes and high specific surface area, the samples demonstrated the remarkable adsorption capacity of methylene blue. The kinetic data were represented by pseudo-second-order kinetic model. Adsorption activation energy was found to be $12.00 \mathrm{~kJ} / \mathrm{mol}$, which were the typical activation energy range for physisorption. The enthalpy change $(\Delta \mathrm{Ho})$ and entropy change $(\Delta \mathrm{So})$ for adsorption are estimated to be $-22.39 \mathrm{~kJ} / \mathrm{mol}$ and $-68.74 \mathrm{~J} / \mathrm{mol} \cdot \mathrm{K}$, respectively. The Gibbs free change $(\Delta \mathrm{Go})$ for the adsorption was estimated to be $-2.245,-1.673,-0.669$ and $-0.296 \mathrm{~kJ} / \mathrm{mol}$ at 293, 303, 313 and 323K, respectively. Therefore, the adsorption was a spontaneous exothermic process.

\section{Acknowledgement}

The authors are grateful for the financial support from the National Natural Science Foundation of China (reference 21106171) and Open fund of Key Laboratory of Comprehensive and Highly Efficient Utilization of Salt Lake Resources.

\section{References}

[1] Niyaz Mohammad Mahmoodi, 2015. Surface modification of magnetic nanoparticle and dye removal from ternary systems, Dyes and Pigments, 27, 251-259

[2] Fu Y, Viraraghavan T. 2011. Fungal decolorization of dye wastewaters a review, Bioresource Technology, 79, 251-262

[3] Jian Zhang, Yan Zhou, Meiyan Jiang, Juan Li, Jiawei Sheng, 2015. Removal of methylene blue from aqueous solution by adsorption on pyrophyllite, Journal of Molecular Liquids, 209, 267-271.

[4] M. Ozacar, I.A. Sengil, 2005. A kinetic study of metal complex dye sorption onto pine sawdust, Process Biochem, 40, 565-572

[5] L. Wang, A.Q. Wang, 2008. Adsorption properties for Congo Red from aqueous solution onto surfactant-modified montmorillonite, J. Hazard. Mater. 160, 173-180.

[6] P. Baskaralingam, M. Pulikesi, V. Ramamurthi, S. Sivanesan, 2006. Equilibrium studies for the adsorption of acid dye onto modified hectorite, J. Hazard. Mater. B136, 989-992.

[7] Duyuan Yue, Yan Jing, Jun Ma, Chenglong Xia, 2011. Removal of Neutral red from aqueous solution by using modified hectorite, Desalination, 267, 9-15.

[8] Chenglong Xia, Yan Jing, Yongzhong Jia, Duyuan Yue, Jun Ma, 2011. Adsorption properties of congo red from aqueous solution on modified hectorite: Kinetic and thermodynamic studies, Desalination, 265, 81-87

[9] Jun Ma, Yongzhong Jia, Yan Jing, Jinhe Sun, 2010. Equilibrium models and kinetic for the adsorption of methylene blue on Co-hectorites, Journal of Hazardous Materials, 175 , 965-969

[10] R. Chaudhuri, F.L. Arbeloa, I.L. Arbeloa, 2000. Spectroscopic characterization of the adsorption of Rhodamine 3B in hectorite, Langmuir 16 , 1285-1291

[11] Sh. Sohrabnezhad, A. Pourahmad, 2010. Comparison absorption of new methylene blue dye in zeolite and nanocrystal zeolite, Desalination, 256, 84-89. 
[12] Mall Indra D, Srivastava Vimal C, Agarwal Nitin K. 2006. Removal of orange-G and methyl violet dyes by adsorption onto bagasse fly ash-Kinetic study and equilibrium isotherm analyses. Dyes and Pigments, 69 , 210-223.

[13] Debnath Sushanta, Chand Ghosh Uday. 2008. Kinetics, isotherm and thermodynamics for $\mathrm{Cr}(\mathrm{III})$ and $\mathrm{Cr}(\mathrm{VI})$ adsorption from aqueous solutions by crystalline hydrous titanium oxide. Journal of Chemical Thermodynamics, 40, 67-77.

[14] Özcan S, Erdem B, Özcan A. 2004. Adsorption of acid blue 193 from aqueous solutionsonto Na-bentonite and DTMA-bentonite, Journal of Colloid and Interface Science, 280, 44-54 